\title{
Relativistic Rotating Electromagnetic Fields
}

\author{
H. Vargas-Rodríguez $\mathbb{D},{ }^{1}$ A. Gallegos $\mathbb{D},{ }^{1}$ M. A. Muñiz-Torres $\mathbb{D},{ }^{1}$ H. C. Rosu $\mathbb{D}{ }^{2}$ \\ and P. J. Domínguez ${ }^{3}{ }^{3}$ \\ ${ }^{1}$ Departamento de Ciencias Exactas y Tecnologia, Centro Universitario de los Lagos, Universidad de Guadalajara, Enrique Daz de \\ León 1144, Col. Paseos de la Montaña, Lagos de Moreno, Jalisco, Mexico \\ ${ }^{2}$ IPICyT, Instituto Potosino de Investigación Científica y Tecnológica, Camino a la Presa San José 2055, Col. Lomas 4a Sección, \\ 78216 San Luis Potosi S.L.P., Mexico \\ ${ }^{3}$ Centro de Investigación y de Estudios Avanzados del Instituto Politecnico Nacional, Av. Instituto Politecnico Nacional 2508, \\ C.P. 07360 Alcaldía Gustavo A. Madero, Ciudad de Mexico, Mexico
}

Correspondence should be addressed to H. Vargas-Rodríguez; hvargas@culagos.udg.mx

Received 24 August 2020; Accepted 3 December 2020; Published 29 December 2020

Academic Editor: Luis Herrera

Copyright (c) 2020 H. Vargas-Rodríguez et al. This is an open access article distributed under the Creative Commons Attribution License, which permits unrestricted use, distribution, and reproduction in any medium, provided the original work is properly cited. The publication of this article was funded by $\mathrm{SCOAP}^{3}$.

In this work, we consider axially symmetric stationary electromagnetic fields in the framework of special relativity. These fields have an angular momentum density in the reference frame at rest with respect to the axis of symmetry; their Poynting vector form closed integral lines around the symmetry axis. In order to describe the state of motion of the electromagnetic field, two sets of observers are introduced: the inertial set, whose members are at rest with the symmetry axis; and the noninertial set, whose members are rotating around the symmetry axis. The rotating observers measure no Poynting vector, and they are considered as comoving with the electromagnetic field. Using explicit calculations in the covariant $3+1$ splitting formalism, the velocity field of the rotating observers is determined and interpreted as that of the electromagnetic field. The considerations of the rotating observers split in two cases, for pure fields and impure fields, respectively. Moreover, in each case, each family of rotating observers splits in two subcases, due to regions where the electromagnetic field rotates with the speed of light. These regions are generalizations of the light cylinders found around magnetized neutron stars. In both cases, we give the explicit expressions for the corresponding velocity fields. Several examples of relevance in astrophysics and cosmology are presented, such as the rotating point magnetic dipoles and a superposition of a Coulomb electric field with the field of a point magnetic dipole.

\section{Introduction}

The Poynting vector can be used to establish the state of motion of the electromagnetic field, for example, consider a charged line distribution on the $x$-axis, moving along this axis, while an observer at rest measures a Poynting vector, a comoving observer measures none; hence, the velocity of the comoving observer can be identified with the velocity of the electromagnetic field. Since the electromagnetic field is a distributed object, a full family of observers measuring no Poynting vector is required to fully characterize the electromagnetic field's motion.

In relativity, a reference frame is an idealization of an observer equipped with some measuring devices in some motion state; consequently, a set of reference frames is described by a time-like congruence, the world lines of a family of observers. In its turn, this congruence is described by a family of unit time-like vectors, the observers' 4-velocity field, $\tau$.

Covariant electromagnetic fields can be classified according to their first invariant as of magnetic, electric, and null types. The second invariant generates the pure and nonpure classes. Except for pure null fields, it is well known that it is always possible to find the reference frame where the Poynting vector vanishes [1]. Mitskievich showed how to find such a reference frame; he proved that, for any nonnull pure field, it is possible to construct a simple bivector with the electromagnetic field tensor; from it, or its Hodge dual-conjugate, 
a unit time-like vector can be extracted. For pure fields, this vector is used to describe the reference frame in which there is only an electric field or a magnetic field, while for nonpure fields, it describes the reference frame in which both the electric and magnetic vectors are parallel to each other $[2,3]$. It follows that generically electromagnetic fields can have their own dynamical state of motion that may entail a plethora of physical effects and applications.

However, rotation and relativity are hard to match with each other [4] because of some puzzling features. In astrophysics, there exists the so-called light cylinder problem, the lack of understanding about what happens in the region where the magnetic field lines of a rigidly rotating pulsar reach the speed of light [4-6]; some authors argue that the magnetic field lines rotate with a speed greater than that of the light beyond this cylinder [7]. The electromagnetic fields have also opened fundamental questions in terms of rotational motion $[8,9]$, and this theory provides a strong support to model several astrophysical objects, such as pulsars $[10,11]$ and active galactic nuclei $[12]$.

In this paper, we consider axially symmetric electromagnetic fields in rotational motion around the symmetry axis. They are characterized not only by the rotation of the reference frame but also by the fact that such fields have an angular momentum density in any other reference frame different from the one comoving with the field. Indeed, this angular momentum has been observed long ago [13]. In particular, if the reference frame is at rest with respect to the rotation axis, the Poynting vector of these rotational fields will be tangent to the closed lines around the rotation axis. According to Jackson [14], one can always associate an angular momentum density to these vectors. An equivalent approach to determine the angular momentum density is based on Nöther's theorem $[15,16]$.

Reference frames are introduced here by means of both a rotational and a nonrotational time-like congruence in the Minkowski spacetime. The velocity four-vector, $\tau$ (the monad field), associated to them is then used to perform the $3+1$ splitting of any four-dimensional vector and in the splitting of the field tensor into its corresponding electric and magnetic fields. The explicit general covariance of the 3 +1 splitting formalism allows the usage of abstract representations of the tensor quantities. It is applied in special relativity when noninertial effects are evaluated without involving any a priori assumption. Therefore, it is also a natural framework in the theory of general relativity.

Hence, two families of observers will be considered herein, namely, the inertial family, at rest with respect to the rotation axis, and the noninertial family in rotational motion with respect to the same axis. Observers in the rotating family measure no Poynting vector and are considered comoving with the electromagnetic field. A causal border known as the light surface appears whenever the electromagnetic field becomes of the pure null type. This light surface divides the spacetime in at least two different regions; in these cases, the noninertial family may split into two complementary subsets, one for each region. With pure electromagnetic fields, these subfamilies are labeled by the indexes $\{I, I I\}$, while for nonpure fields by $\{A, B\}$. No label is used for iner- tial observers. The two families of observers can be conveniently discussed by using the theory of arbitrary reference frames, also known as the $3+1$ decomposition, orthogonal splitting, and/or the old monad formalism $[15,17,18]$.

The rest of the paper is organized as follows. Section 2 presents the electrodynamics in arbitrary reference frames, the classification of electromagnetic fields in terms of its invariants, and the propagation of electromagnetic fields from the point of view of the electromagnetic field tensor's invariants. Section 3 introduces stationary electromagnetic fields with angular momentum; they are discussed from the point of view of the inertial observers. Section 4 deals with pure rotating electromagnetic fields described from the point of view of both the inertial and the comoving rotating observers; the velocity fields of the last ones are determined with respect to the inertial observers. Section 5 presents some examples regarding the pure rotating fields of point magnetic dipoles, which are generically important models of magnetospheres in both astrophysics and geophysics [19-23]. In Section 6, the corresponding 4-velocity field of comoving observers with nonpure electromagnetic fields is presented. Section 7 shows an example of an impure field, the superposition of an electric Coulomb field, and the magnetic field of a point dipole. Section 8 presents the conclusions. There are also two Appendixes, one on the basic definition of the Car$\tan$ formalism and the theory of arbitrary reference frames and a second one which gives the expressions of the charge and current densities in a rotating reference frame.

Here, we use the signature $(+,-,-,-)$ and a system of units in which $c=1$ (unless explicitly shown otherwise for convenience). Greek indices are taken to run from 0 to 3 and Latin indices from 1 to 3 , and we adopt the standard convention for summation over repeated indices. Furthermore, we will indicate three vectors with bold symbols.

\section{Electromagnetic Fields in Arbitrary Reference Frames}

In general, field tensors $F$ in arbitrary reference frames are written as 2-form:

$$
F=\frac{1}{2} F_{\mu \nu} d x^{\mu} \wedge d x^{\nu}
$$

On the other hand, the electromagnetic vector potential is written as a covector, $A=A_{\mu}(x) d x^{\mu}$, and the connection between $F$ and $A$ is given by the relationship:

$$
F=d A
$$

With respect to a given reference frame, the field tensor splits into two terms:

$$
F=\mathbf{E} \wedge \tau+*(\mathbf{B} \wedge \tau)
$$

involving the electric and magnetic covectors [15]. The * operation denotes the Hodge dual conjugation defined in Equation (A.3). 
From a given electromagnetic field tensor, the corresponding electric and magnetic covectors in the reference frame represented by the monad field $\tau$ can be calculated from the following:

$$
\begin{aligned}
& \mathbf{E}=*(\tau \wedge * F), \\
& \mathbf{B}=*(\tau \wedge F) .
\end{aligned}
$$

The Poynting vector can be written as a Hodge conjugate as well as the following:

$$
\mathbf{S}=\frac{\mathbf{E} \times \mathbf{B}}{4 \pi}=\frac{1}{4 \pi} *(\mathbf{E} \wedge \tau \wedge \mathbf{B}) .
$$

See (A.10).

An arbitrary vector can be decomposed into the sum of a vector parallel to $\tau$, the time-like component, and a vector perpendicular to $\tau$, which is the space-like component. For example, as well known, the four-vector of the electric current can be written in terms of the four-dimensional velocity $u^{\mu}$ and decomposed as follows:

$$
\begin{gathered}
j^{\mu}=\rho u^{\mu}=\rho \tau^{\mu}+{\stackrel{(3)}{\mathbf{j}^{\mu}}}, \\
\stackrel{(\tau)}{\rho}=j \cdot \tau, \\
\stackrel{(3)}{\mathbf{j}^{\mu}}=b^{\mu v} j_{v},
\end{gathered}
$$

where $\rho^{(\tau)}$ and $\mathbf{j}^{\mu(3)}$ are the charge and current densities measured in the reference frame represented by the monad field $\tau$ , respectively, while $b_{\mu \nu}$ is the four-dimensional projector; $b$ $=g-\tau \otimes \tau$ is also used to define the three-dimensional scalar product of the differential forms denoted by $\bullet$ in the following (see Appendix A).

The electromagnetic splitting (Equation (3)), with Equation (4), is the consequence of the component form of the Lorentz force [15]:

$$
\mathbf{f}_{L}=(\mathbf{E}+\mathbf{v} \times \mathbf{B})_{\alpha}=F_{\mu \nu}\left(\tau^{v}+\mathbf{v}^{v}\right) b_{\alpha}^{\mu} .
$$

Here, the three-dimensional velocity $\mathbf{v}$ of the charged particle acted by the Lorentz force follows from the general definition:

$$
u=\stackrel{(\tau)}{u}(\tau+\mathbf{v}) \Rightarrow \mathbf{v}=b\left(\frac{d x}{d s}, \cdot\right)
$$

where $u^{(\tau)}=u \cdot \tau=d t / d s=\left(1-v^{2}\right)^{-1 / 2}$, while $d t=\tau_{\mu} d x^{\mu}=\tau$ $\cdot d x$.

2.1. Classification of Electromagnetic Fields and Its Propagation. The classification of electromagnetic fields is based on their invariants (see $[1,3])$ :

$$
\begin{gathered}
I_{1}=-2 *(F \wedge * F)=F_{\mu \nu} F^{\mu \nu}=2(\mathbf{B} \bullet \mathbf{B}-\mathbf{E} \bullet \mathbf{E}), \\
I_{2}=2 *(F \wedge F)=F_{\mu \nu}^{*} F^{\mu \nu}=4 \mathbf{E} \bullet \mathbf{B},
\end{gathered}
$$

where

$$
F_{\mu \nu}^{*}=\frac{1}{2} E_{\mu \nu \sigma \tau} F^{\sigma \tau}
$$

The first invariant suggests that the fields can be classified according to their sign in the following kinds: (1) magnetic type if $I_{1}>0$, (2) electric type if $I_{1}<0$, and (3) null type if $I_{1}=0$. On the other hand, the second invariant allows to introduce an additional subclassification in (a) pure if $I_{2}=0$ and (b) nonpure if $I_{2} \neq 0$.

Some useful identities are as follows:

$$
\begin{gathered}
F_{\alpha \gamma} F^{\beta \gamma}-F_{\alpha \gamma}^{*} F_{*}^{\beta \gamma}=\frac{1}{2} I_{1} \delta_{\gamma}^{\beta}, \\
F_{\alpha \gamma}^{*} F^{\beta \gamma}=\frac{1}{4} I_{2} \delta_{\gamma}^{\beta} .
\end{gathered}
$$

We move now to the deduction of an important result [3]; connecting the propagation speed of the electromagnetic field with the electromagnetic field invariants is presented. Consider the electromagnetic energy momentum tensor as follows:

$T_{v}^{\mu}=-\frac{1}{4 \pi}\left[F^{\mu \alpha} F_{v \alpha}-\frac{1}{4} F_{\sigma \tau} F^{\sigma \tau} \delta_{v}^{\mu}\right]=-\frac{1}{4 \pi}\left[F^{\mu \alpha} F_{v \alpha}-F_{*}^{\mu \alpha} F_{v \alpha}^{*}\right]$,

in Gaussian units. When it is contracted with an arbitrary monad, it contains the electromagnetic energy density and the Poynting vector in the corresponding reference frame:

$$
T_{v}^{\mu} \tau^{v}=\frac{1}{8 \pi}\left[\left(\mathbf{B}^{2}+\mathbf{E}^{2}\right) \tau_{v}-2(\mathbf{E} \times \mathbf{B})_{v}\right]
$$

and the squared expression is calculated using Equation (11):

$$
\begin{aligned}
T_{v}^{\mu} T_{\mu \lambda} \tau^{\nu} \tau^{\lambda}= & \frac{1}{(8 \pi)^{2}}\left[\left(\mathbf{B}^{2}+\mathbf{E}^{2}\right)^{2}-4(\mathbf{E} \times \mathbf{B})^{2}\right] \\
& =\frac{1}{(8 \pi)^{2}}\left[\left(\mathbf{B}^{2}-\mathbf{E}^{2}\right)^{2}+4(\mathbf{E} \bullet \mathbf{B})^{2}\right]=\frac{1}{(16 \pi)^{2}}\left(I_{1}^{2}+I_{2}^{2}\right)
\end{aligned}
$$

These constructions are not only scalars under coordinate transformations, but they are also independent of the choice of reference frame since the right hand side does not depend on the monad.

Landau and Lifshitz [1] have shown that the propagation speed of the electromagnetic field is given by the following:

$$
\frac{\mathbf{v}}{1+\mathbf{v}^{2}}=\frac{\mathbf{E} \times \mathbf{B}}{\mathbf{E}^{2}+\mathbf{B}^{2}}
$$


Using Equation (15), Mitskievich has shown that the propagation speed of electromagnetic fields satisfies the following:

$$
0 \leq \frac{|\mathbf{v}|}{1+\mathbf{v}^{2}}=\frac{1}{2} \sqrt{1-\frac{I_{1}^{2}+I_{2}^{2}}{4\left(\mathbf{E}^{2}+\mathbf{B}^{2}\right)^{2}}}=\frac{|\mathbf{E}||\mathbf{B}|}{\mathbf{E}^{2}+\mathbf{B}^{2}}|\sin \psi| \leq \frac{1}{2} .
$$

Hence, only null pure fields do propagate with the speed of light. Equation (16) is valid for observers with arbitrary motion on the background of curved spacetime; it coincides with Equation (15) when inertial observers in flat spacetime are considered.

2.2. Covariant Maxwell Equations. The covariant dynamical and constitutive Maxwell equations are as follows:

$$
\begin{gathered}
\delta F=4 \pi j \Leftrightarrow F_{; \nu}^{\mu \nu}=-4 \pi j^{\mu}, \\
d F=0 \Leftrightarrow F_{[\alpha \beta, \gamma]=0},
\end{gathered}
$$

where $\delta=-* d *$

Taking into account that the divergence and curl operators in an arbitrary reference frame have the forms $\operatorname{div} \mathbf{V}=$ $-* d * \mathbf{V}-\mathbf{G} \bullet \mathbf{E}$ and curl $\mathbf{V}=*(\tau \wedge d \mathbf{V})$, the $3+1$ splitted form of Maxwell's equations is as follows [15]:

$$
\operatorname{div} \mathbf{E}=4 \pi \stackrel{(\tau)}{\rho}+2 \omega \bullet \mathbf{B}
$$

$$
\begin{gathered}
\operatorname{curl} \mathbf{B}+\mathbf{G} \times \mathbf{B}=\left(£_{\tau} \mathbf{E}-2 \mathbf{E}_{v} D_{\mu}^{v} d x^{\mu}+D_{\alpha}^{\alpha} \mathbf{E}\right)+4 \pi \stackrel{(3)}{\mathbf{j}} \\
\qquad \operatorname{div} \mathbf{B}=-2 \omega \bullet \mathbf{E} \\
\operatorname{curl} \mathbf{E}+\mathbf{G} \times \mathbf{E}=-\left(£_{\tau} \mathbf{B}-2 \mathbf{B}_{v} D_{\mu}^{v} d x^{\mu}+D_{\alpha}^{\alpha} \mathbf{B}\right)
\end{gathered}
$$

and $£_{\tau}$ is the Lie derivative with respect to $\tau[24,25]$. These are the electromagnetic equations which should be used by the observers in motion defined by the $\tau$ congruence. The additional terms in Maxwell's equations are interpreted as the charge and current densities (both electric and magnetic ones) of kinematic or fictitious nature, the latter by analogy with the fictitious forces which appear in noninertial frames [15].

When the covariant Maxwell equations are considered in their orthogonal $3+1$ splitting form, the following remarks can be brought out:

(i) From Gauss' law (Equation (19)), it follows that for a pure magnetic field, $(\mathbf{E}=0)$, an electric charge density should always exist in order to compensate the kinematic (fictitious) charge induced by the rotation of the magnetic field

(ii) From Ampere's law (Equation (20)), it follows that for a pure electric field, $(\mathbf{B}=0)$, there should always exist an electric current density to compensate the kinematic (fictitious) current induced by the defor- mation of the reference frame and/or the nonstationarity of the electric field

(iii) For pure electric fields, the law of absence of monopoles (Equation (21)) requires that the electric field should be orthogonal to the angular velocity $\omega$. There should be no kinematic monopole densities

(iv) On the other hand, Faraday's induction law (Equation (22)) for pure magnetic fields requires the nonexistence of kinematic magnetic current densities

(v) In both cases, Equations (21) and (22) as originating from the constitutive Maxwell equations will be satisfied whenever the field tensor is an exact form, $F$ $=d A$, because $d F=0$ and $d d A \equiv 0$

\section{Electromagnetic Fields with Angular Momentum}

The four-potential of a stationary axially symmetric field is as follows:

$$
A=M(r, \vartheta) d t-N(r, \vartheta) d \varphi
$$

The corresponding electromagnetic field has the following expression:

$F=\left(M_{, r} \theta^{(1)}+\frac{1}{r} M_{, \vartheta} \theta^{(2)}\right) \wedge \theta^{(0)}-\frac{1}{r \sin \vartheta}\left(N_{, r} \theta^{(1)}+\frac{1}{r} N_{, \vartheta} \theta^{(2)}\right) \wedge \theta^{(3)}$,

and the electric and magnetic fields (Equation (4)), with respect to an inertial reference frame represented by $\tau=\theta^{(0)}$, are as follows:

$$
\begin{gathered}
\mathbf{E}=M_{, r} \theta^{(1)}+\frac{1}{r} M_{, \vartheta} \theta^{(2)}, \\
\mathbf{B}=-\frac{1}{r \sin \vartheta}\left(\frac{1}{r} N_{, \vartheta} \theta^{(1)}-N_{, r} \theta^{(2)}\right) .
\end{gathered}
$$

The Poynting covector, in this inertial reference frame, is as follows:

$$
\mathbf{S}=-\frac{1}{4 \pi r \sin \vartheta}\left(M_{, r} N_{, r}+\frac{1}{r^{2}} M_{, \vartheta} N_{, \vartheta}\right) \theta^{(3)} .
$$

It can be seen that it points in the azimuthal direction; hence, according to Jackson [14], the fields (Equation (23)) possess an angular momentum density.

From Maxwell's Equation (17), the 4-current covector is readily calculated, $j=\rho \theta^{(0)}+\mathrm{J} \theta^{(3)}$, where the charge density measured in the inertial reference frame is as follows:

$$
\rho=-\frac{1}{4 \pi}\left[\frac{1}{r^{2}} \frac{\partial}{\partial r}\left(r^{2} \frac{\partial M}{\partial r}\right)+\frac{1}{r^{2} \sin \vartheta} \frac{\partial}{\partial \vartheta}\left(\sin \vartheta \frac{\partial M}{\partial \vartheta}\right)\right],
$$


while the corresponding current density is as follows:

$$
\mathbf{J}=\frac{1}{4 \pi}\left[\frac{1}{r \sin \vartheta} \frac{\partial^{2} N}{\partial r^{2}}+\frac{1}{r^{3}} \frac{\partial}{\partial \vartheta}\left(\frac{1}{\sin \vartheta} \frac{\partial N}{\partial \vartheta}\right)\right] .
$$

The corresponding expressions for a rotating reference frame can be easily found by inserting Equations (28) and (29) into Equation (B.4).

For the fields under consideration (Equation (23)), the electromagnetic invariants are as follows:

$$
\begin{gathered}
I_{1}=2\left[\frac{1}{r^{2} \sin ^{2} \vartheta}\left(N_{, r}^{2}+\frac{1}{r^{2}} N_{, \vartheta}^{2}\right)-\left(M_{, r}^{2}+\frac{1}{r^{2}} M_{, \vartheta}^{2}\right)\right], \\
I_{2}=\frac{4}{r^{2} \sin \vartheta}\left(M_{, \vartheta} N_{, r}-M_{, r} N_{, \vartheta}\right) .
\end{gathered}
$$

Electromagnetic fields of many different types may be considered according to the choices of the functions $M(r, \vartheta$ ) and $N(r, 9)$. Pure electromagnetic rotating fields are obtained when either $M=M(N)$ or $N=N(M)$. The first case corresponds to pure magnetic rotating fields, while the second to a pure electric rotating fields, but only the first case is considered here, since the second one is quite similar. The cases $M=0$ or $N=0$ result in fields with vanishing Poynting vector. Impure rotating fields correspond to other choices of $M$ and $N$.

\section{Pure Rotating Electromagnetic Fields}

We consider the special case when $M=M(N(r, \vartheta))$. In this case, the electromagnetic field tensor is a simple bivector:

$$
\begin{aligned}
F & =\frac{d M}{d N}\left(N_{, r} \theta^{(1)}+\frac{1}{r} N_{, \vartheta} \theta^{(2)}\right) \wedge\left(\theta^{(0)}-\frac{1}{\xi} \theta^{(3)}\right) \\
& =-*\left[\frac{1}{r \sin \vartheta}\left(\frac{1}{r} N_{, \vartheta} \theta^{(1)}-N_{, r} \theta^{(2)}\right) \wedge\left(\theta^{(0)}-\xi \theta^{(3)}\right)\right],
\end{aligned}
$$

where we have introduced the notation:

$$
\xi(r, \vartheta)=\frac{d M}{d N} r \sin \vartheta
$$

For the special case considered here, it is possible to see from Equations (25) and (26) that in the inertial reference frame, several electric fields,

$$
\mathbf{E}=\frac{d M}{d N}\left(N_{, r} \theta^{(1)}+\frac{1}{r} N_{, \vartheta} \theta^{(2)}\right),
$$

can be associated to the same magnetic field:

$$
\mathbf{B}=-\frac{1}{r \sin \vartheta}\left(\frac{1}{r} N_{, \vartheta} \theta^{(1)}-N_{, r} \theta^{(2)}\right) .
$$

Of course, in the alternative case, $N=N(M)$, several magnetic fields can be associated to the same electric field. The fields (Equation (33)) are unipolar-induced electric fields by the rotation of the magnetic field. As it would be seen below, the derivative $d M / d N$ is interpreted as the angular speed of the magnetic field.

The first electromagnetic field invariant becomes the following:

$$
I_{1}=\frac{2}{r^{2} \sin ^{2} \vartheta}\left(N_{, r}^{2}+\frac{1}{r^{2}} N_{, \vartheta}^{2}\right)\left(1-\xi^{2}\right) .
$$

For $|\xi|<1$, it is of pure magnetic type, while for $|\xi|=1$, it is of pure null type; if $|\xi|>1$, it is of the pure electric type. In this sense, $\xi$ from Equation (32) divides Minkowski spacetime into different regions.

In the spacetime regions where $|\xi|<1$, we can choose the monad field for the vanishing Poynting observers as follows:

$$
\tau_{I}=\frac{1}{\sqrt{1-\xi^{2}}}\left(\theta^{(0)}-\xi \theta^{(3)}\right) .
$$

Comparing Equation (36) with Equation (A.14), one finds the velocity field of the comoving observers with the electromagnetic field in the inertial reference frame given by the following:

$$
v_{I}(r, \vartheta)=\xi=\frac{d M}{d N} r \sin \vartheta .
$$

Consequently, as it was mentioned, the derivative $d M / d$ $N$ may be interpreted as the angular speed of the observers $I$ and therefore as that of the magnetic field. It is as follows:

$$
\Omega_{I}(r, \vartheta)=\frac{d M}{d N}(r, \vartheta) .
$$

The electromagnetic field tensor (Equation (31)) in terms of the monad field (Equation (36)) is as follows:

$$
F=-*\left[\frac{\sqrt{1-\xi^{2}}}{r \sin \vartheta}\left(\frac{1}{r} N_{, \vartheta} \theta^{(1)}-N_{, r} \theta^{(2)}\right) \wedge \tau_{I}\right] .
$$

In the frame (Equation (36)), the electric field vanishes and only the magnetic field vector remains and can be obtained from comparing Equation (39) with Equation (3):

$$
\mathbf{B}_{I}=-\frac{\sqrt{1-\xi^{2}}}{r \sin \vartheta}\left(\frac{1}{r} N_{, \vartheta} \theta^{(1)}-N_{, r} \theta^{(2)}\right),
$$

while the Poynting vector vanishes in this reference frame.

For the spacetime regions where $|\xi|>1$, we can choose the monad field for the vanishing Poynting observers as the following:

$$
\tau_{I I}=\frac{|\xi|}{\sqrt{\xi^{2}-1}}\left(\theta^{(0)}-\frac{1}{\xi} \theta^{(3)}\right) .
$$


Comparing Equation (41) with Equation (A.14), one can find the velocity field of the comoving observers with the electromagnetic field in the inertial reference frame:

$$
v_{I I}(r, \vartheta)=\frac{1}{\xi(r, \vartheta)}
$$

The electromagnetic field tensor (Equation (31)) in terms of the monad field (Equation (41)) is as follows:

$$
F=\frac{\sqrt{\xi^{2}-1}}{|\xi|} \frac{d M}{d N}\left(N_{, r} \theta^{(1)}+\frac{1}{r} N_{, \vartheta} \theta^{(2)}\right) \wedge \tau_{I I} .
$$

In the frame represented by the monad field (Equation (41)), the magnetic field vanishes and the surviving electric field vector can be obtained by comparing Equation (43) with Equation (3):

$$
\mathbf{E}_{I I}=\frac{\sqrt{\xi^{2}-1}}{|\xi|} \frac{d M}{d N}\left(N_{, r} \theta^{(1)}+\frac{1}{r} N_{, \vartheta} \theta^{(2)}\right)
$$

and the Poynting vector vanishes in this reference frame.

The two congruences, defined by the monad fields (Equations (36) and (41)), are separated by the light surface, which is the surface where the first electromagnetic invariant vanishes. On this surface, the electromagnetic field rotates with the speed of light; the field tensor (Equation (31)) is the exterior product of a null covector and a spatial covector. Consequently, it is not possible to find a reference frame rotating in synchrony with the electromagnetic field, as expected from Mitskievich's Equation (16).

\section{Point Magnetic Dipoles in an Arbitrary Rotation State}

Rotating point magnetic dipoles are special cases of pure electromagnetic fields. They provide common models of the magnetospheres of planets and stars $[19,22]$. In order to consider them, the following function is used:

$$
N(r, \vartheta)=\frac{k \sin ^{2} \vartheta}{r}
$$

In the inertial reference frame at rest with the rotation axis, the electric and magnetic covectors, the Poynting covector, the charge density, and the three-dimensional current density, are found, inserting Equation (45) in Equations (25), (26), (27), (28) and (29):

$$
\begin{gathered}
\mathbf{E}=-k \frac{d M}{d N}\left(\frac{\sin ^{2} \vartheta}{r^{2}} \theta^{(1)}-\frac{2 \sin \vartheta \cos \vartheta}{r^{2}} \theta^{(2)}\right), \\
\mathbf{B}=-k\left(\frac{2 k \cos \vartheta}{r^{3}} \theta^{(1)}+\frac{k \sin \vartheta}{r^{3}} \theta^{(2)}\right), \\
\mathbf{S}=-\frac{k^{2} \sin \vartheta}{4 \pi r^{5}}\left(\frac{d M}{d N}\right)\left(\sin ^{2} \vartheta+4 \cos ^{2} \vartheta\right) \theta^{(3)},
\end{gathered}
$$

$$
\rho=\frac{k}{4 \pi r^{2}}\left[\sin ^{2} \vartheta \frac{\partial}{\partial r}\left(\frac{d M}{d N}\right)-\frac{1}{r \sin \vartheta} \frac{\partial}{\partial \vartheta}\left(\sin ^{2} \vartheta \cos \vartheta \frac{d M}{d N}\right)\right],
$$

$$
\mathbf{J}=0
$$

The magnetic field corresponds to that of a point dipole of magnitude $|k|$ aligned with the $z$-axis. But for each choice of $M(N)$, there is a different electric field, though they differ up to a conformal factor. The corresponding Poynting vectors differ also in the same manner. As seen before, the derivative $d M / d N$ is connected with the rotation of the electromagnetic field (Equation (38)). The charge density structure is now dictated by this derivative, giving rise to a vast number of possibilities; see some examples below. The function $M(N)$, or its derivative, has to be chosen from physical considerations or from observations; for example, from an observed charge density, it should be possible to reconstruct the derivative by solving the differential Equation (49).

In this case, the first invariant becomes the following:

$$
I_{1}=\frac{2 k^{2}}{r^{6}}\left(\sin ^{2} \vartheta+4 \cos ^{2} \vartheta\right)\left(1-\xi^{2}\right)
$$

with $\xi$ given by Equation (32). Notice that on the light surface $|\xi|=1$, the first invariant vanishes. However, the electric and magnetic fields, Equations (46) and (47), respectively, are everywhere well defined. This light surface is the boundary where the electric and magnetic fields have the same strength.

In the region $|\xi|<1$, the electromagnetic field is of the pure magnetic type; rotating observers described by the monad field (Equation (36)) detect only a dipolar-like magnetic field:

$$
\mathbf{B}_{\mathbf{I}}=-k \sqrt{1-\xi^{2}}\left(\frac{2 \cos \vartheta}{r^{3}} \theta^{(1)}+\frac{\sin \vartheta}{r^{3}} \theta^{(2)}\right) .
$$

In the region $|\xi|>1$, the electromagnetic field is of the pure electric type, and the rotating observers described by the monad field (Equation (41)) detect only an electric field given by the following:

$$
\mathbf{E}_{\mathrm{II}}=k \frac{\sqrt{\xi^{2}-1}}{|\xi|} \frac{d M}{d N}\left(\frac{\sin ^{2} \vartheta}{r^{2}} \theta^{(1)}-\frac{2 \sin \vartheta \cos \vartheta}{r^{2}} \theta^{(2)}\right)
$$

In the following sections, some illustrative examples of rotating point magnetic dipoles are presented. They show the rich structure of their magnetospheres and give an idea of how different they may be. It is interesting that the charge density shows regions of different polarities, depending on whether the angular velocity and the magnetic moment are parallel or antiparallel. These regions of definite sign charge offer a safe environment for antimatter to accumulate and in principle could be used to explain the antiprotons found in Earth's magnetosphere; see [26, 27]. In addition, the electromagnetic field's rotation velocities are provided and the light surfaces are presented for each case. 
5.1. Point Magnetic Dipoles with Rigid Rotation. To consider a rotating point magnetic dipole in rigid rotation, we use the following:

$$
\Omega_{I}(r, \vartheta)=\frac{d M}{d N}=\Omega_{0}=\text { const. }
$$

which plugged in Equations (37) and (42) leads to the following:

$$
\begin{aligned}
v_{I} & =\Omega_{0} r \sin \vartheta \\
v_{I I} & =\frac{1}{\Omega_{0} r \sin \vartheta} .
\end{aligned}
$$

Hence, only comoving observers I move rigidly, while comoving observers $I I$, outside the light cylinder, move with a subluminal speed; see Figure 1. Thus, at most, the electromagnetic field reaches the speed of light on this light surface; see Figure 2(a). These results counter the idea that the electromagnetic fields do rotate with a superluminal speed outside of the light cylinder; see Fendt and Ouyed [7]. Notice that the speeds of the reference frames $I$ and $I I$ were derived from the very electromagnetic field tensor (Equation (31)) assuming only a dipolar magnetic field nature (Equation (45)) and rigid rotation (Equation (54)).

The rigid dipole is not isolated as there are clouds of charge of different signs around it. In the inertial reference frame, this charge is as follows:

$$
\rho=\frac{\Omega_{0} k}{2 \pi r^{3}}\left(3 \sin ^{2} \vartheta-2\right)
$$

which is well defined on the light cylinder and beyond it. If the angular velocity and the dipolar moment are parallel to each other, there are two lobular clouds of positive charge and one toroidal cloud of negative charge, while if they are antiparallel, the signs of the clouds become inverted. Figure 3(a) shows some revolution profiles of constant charge density for $\Omega_{0}=10$ and $k=1$, in suitable units. These clouds of charges are similar to that found in numerical studies of pulsar magnetospheres; see Kalapotharakos et al. [28] and Cerutti and Beloborodov [29]. It is also a special case of the charge density found by Hones and Bergeson [23]. Kalapotharakos et al. found numerically that the pulsar magnetosphere can be extended well beyond the light cylinder.

5.2. Point Magnetic Dipoles with Differential Rotation and a Deformed Light Cylinder. As a toy model of a differentially rotating magnetic dipole, consider the following angular speed:

$$
\Omega_{I}(r, \vartheta)=\frac{d M}{d N}=\Omega_{0} e^{-\mathrm{aN}(r, \vartheta)}
$$

where $N(r, \vartheta)$ is given by Equation (45), and $a$ and $\Omega_{0}$ are constants. Here, $\Omega_{I}$ is a positive bounded function, and no other physical criteria will be taken into account.

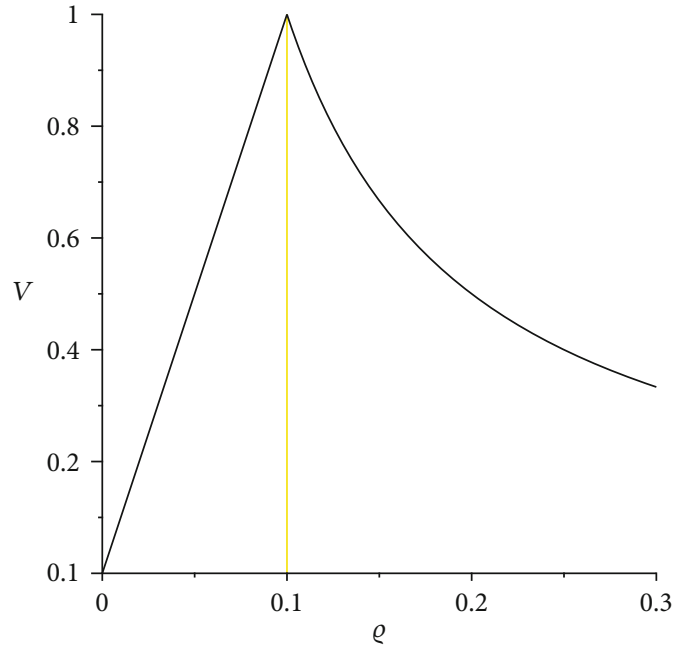

FIgure 1: Speed of the vanishing Poynting vector observers for a rigidly rotating magnetic dipole. Inside the light cylinder, $\mathrm{Q}<0.1$, the electromagnetic field is of the pure magnetic type and rotates rigidly with a subluminal speed $v<1$, on the light cylinder, $\varrho=0.1$, the field is of the pure null type and rotates with the speed of light, and outside the light cylinder, $\varrho>0.1$, the electromagnetic field is of the pure electric type and rotates differentially with a subluminal speed. Here, $\mathrm{Q}$ is the cylindrical radial coordinate.

Substituting Equation (57) in Equations (37) and (42) leads to the following:

$$
\begin{aligned}
& v_{I}=\Omega_{0} e^{-a k \sin ^{2} \vartheta / r} r \sin \vartheta \\
& v_{I I}=\frac{1}{\Omega_{0} r \sin \vartheta} e^{a k \sin ^{2} \vartheta / r} .
\end{aligned}
$$

$v_{I}<1$ is inside the light surface, which is a deformed cylinder, while $v_{I I}<1$ is outside it. Figure 2(b) shows a revolution profile for the light surface corresponding to $v_{I}=1$ when the values $a=1, k=1$, and $\Omega_{0}=10$, in some suitable units, are used.

In this case, the charge density measured in the reference frame at rest with the rotation axis is as follows:

$\rho=-\frac{k \Omega_{0}}{4 \pi r^{4}}\left[3 a k \cos ^{4} \vartheta+(6 r-2 a k) \cos ^{2} \vartheta-2 r-a k\right] e^{-a k \sin ^{2} \vartheta / r}$.

Figure 3(b) shows some revolution profiles for surface of constant density charge, for $a=1, k=1$, and $\Omega_{0}=10$. When the dipolar moment and the angular velocity are antiparallel, the signs of the charge of these surfaces become interchanged.

5.3. Point Magnetic Dipoles with Differential Rotation and a Light-Like Torus. Another possible choice, using the same requirement as before, is as follows:

$$
\Omega_{I}(r, \vartheta)=\frac{d M}{d N}=\frac{\Omega_{0}}{N(r, \vartheta)^{2}} e^{-\mathrm{aN}(r, \vartheta)^{-2}},
$$



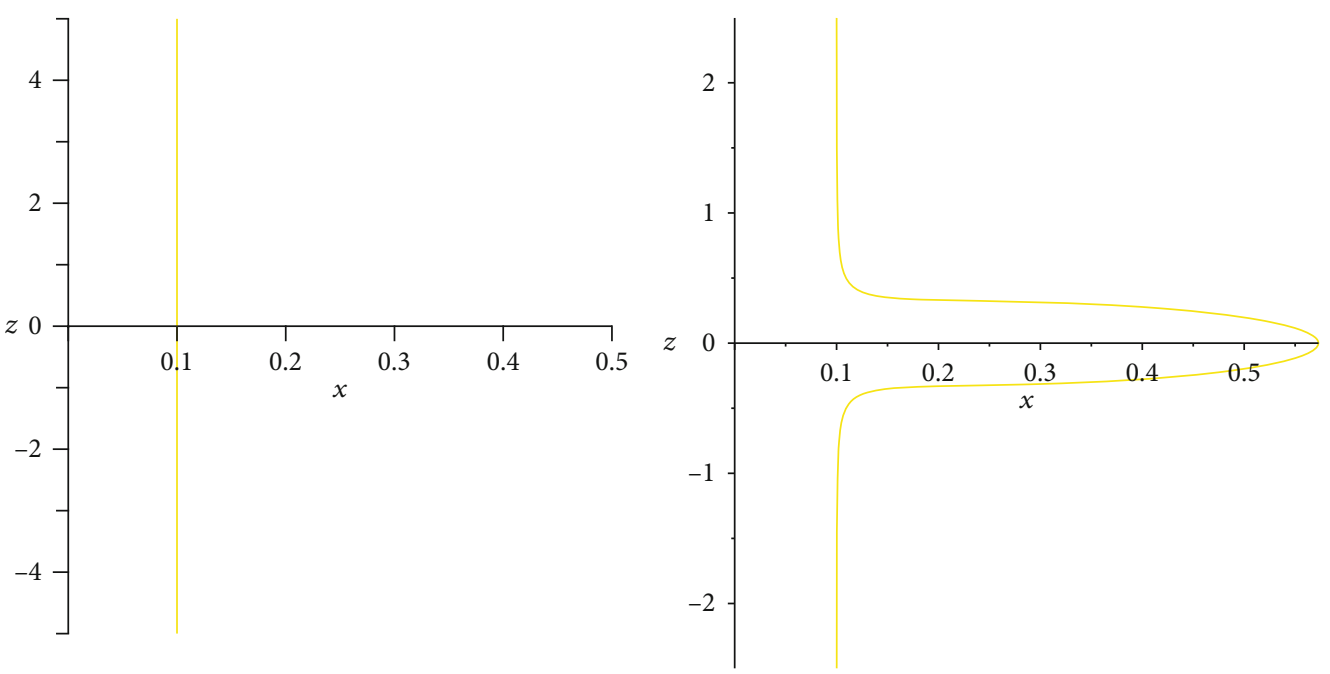

(a)

(b)

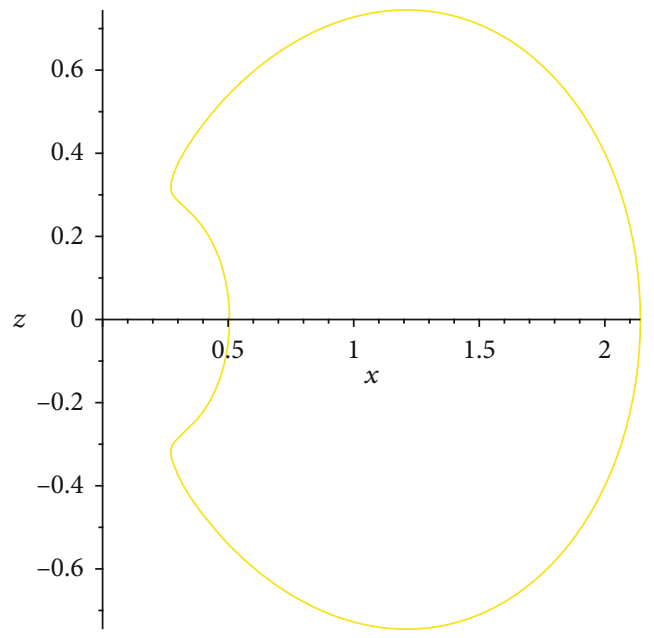

(c)

FIGURE 2: Revolution profiles around the vertical axis of light surfaces, $v=1$, corresponding, respectively, to Equations (55), (58), and (61). On these surfaces, both electromagnetic invariants vanish and the electromagnetic field is rotating with the speed of light in agreement with Equation (16). Hence, two different sets of corotating observers are required on each side to describe the motion of the electromagnetic field. (a) The light cylinder. (b) A cylinder with an equatorial bulge. (c) A light torus is displayed. The electromagnetic field is of the pure magnetic type inside the light surfaces (a) and (b) and of the pure electric type outside of them; this situation is inverted in (c). Figures were plotted using $a=1, k=1$, and $\Omega_{0}=10$.

where $N(r 9)$ is given by Equation (45). Substituting Equation (60) in Equations (37) and (42) leads to the following:

$$
\begin{gathered}
v_{I}=\frac{\Omega_{0} r^{3}}{k^{2} \sin ^{3} \vartheta} e^{-a r^{2} / k^{2} \sin ^{4} \vartheta}, \\
v_{I I}=\frac{k^{2} \sin ^{3} \vartheta}{\Omega_{0} r^{3}} e^{a k \sin ^{2} \vartheta / r} .
\end{gathered}
$$

$v_{I}<1$ is outside the light surface, in this case a torus-like surface, while $v_{I I}<1$ is inside it; the light surface is shown in Figure 2(c). The field is of pure magnetic type outside this torus and of pure electric type inside it.
In the inertial reference frame, at rest with the rotation axis, the charge density is as follows:

$$
\rho=\frac{a \Omega_{0}}{2 \pi r k^{3} \sin ^{8} \vartheta}\left[2 k^{2} \cos ^{4} \vartheta-\left(3 a r^{2}+4 k^{2}\right) \cos ^{2} \vartheta-a r^{2}-2 k^{2}\right] e^{-a r^{2} / k^{2} \sin ^{4} \vartheta} .
$$

Some revolution profiles of constant charge density are shown in Figure 3(c), using $a=1, k=1$, and $\Omega_{0}=10$.

\section{Nonpure Rotating Electromagnetic Fields}

To find the reference frame comoving with the electromagnetic field, one looks for the frame in which the Poynting vector vanishes, i.e., that in which the electric and magnetic 


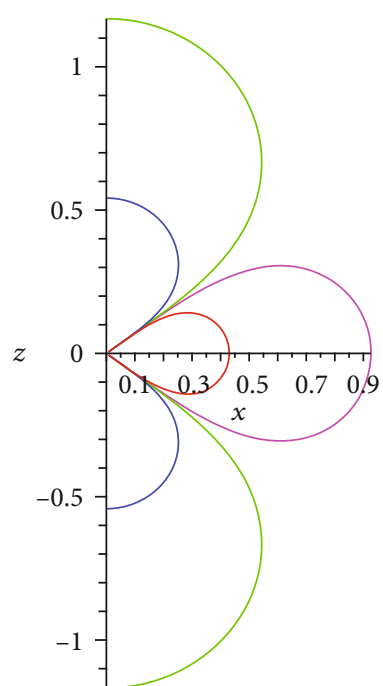

(a)

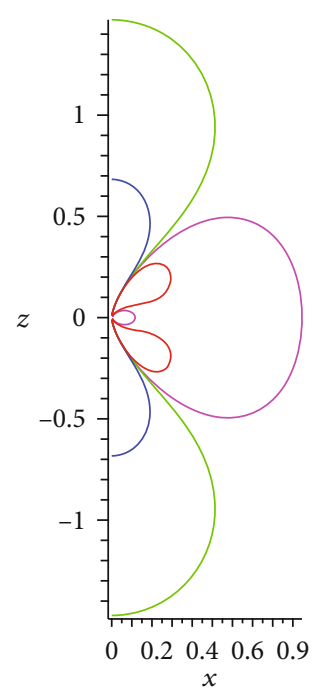

(b)

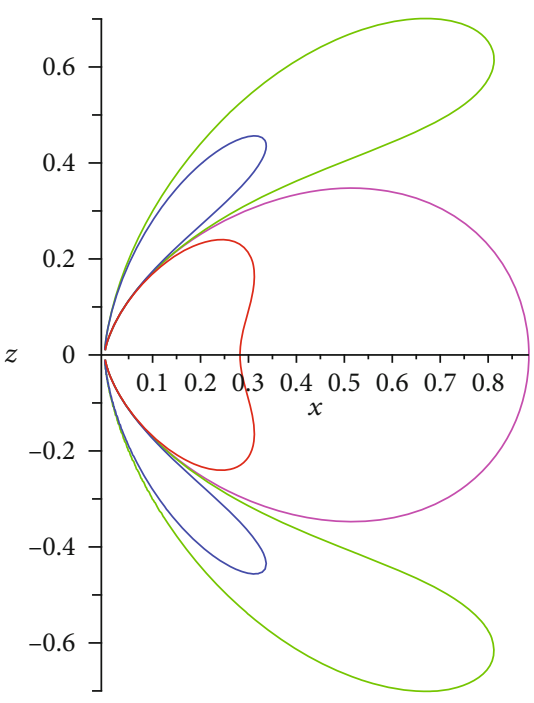

(c)

Figure 3: Contours of revolution surfaces around the vertical axis of constant charge density measured in the reference frame at rest with the vertical axis, on the left for a rigid rotating magnetic dipole (Equation (56)); the cases of differential rotation are shown on the center and left, respectively, for charge densities (Equations (59) and (62)). The green curve corresponds to $\rho=-1$, blue is for $\rho=-10$, red for $\rho=+10$, and magenta for $\rho=+1$. These examples suggest that magnetospheres around astrophysical objects may be quite diverse. Figures were plotted using $a=1, k=1$, and $\Omega_{0}=10$.

fields are parallel to each other. To achieve this goal, we introduce an auxiliar 2-form; see Mitskievich [3]:

$$
\mathcal{F}=\cos (\alpha) \mathcal{F}+\sin (\alpha) * \mathcal{F}
$$

where $\alpha(x)$ is an arbitrary function chosen conveniently.

The original electromagnetic field tensor can be readily obtained from the following:

$$
F=\cos (\alpha) \mathcal{F}-\sin (\alpha) * \mathcal{F} .
$$

Using the auxiliary 2-form, we construct the auxiliary invariants:

$$
\begin{gathered}
\mathcal{I}_{1}=-2 *(\mathcal{F} \wedge * \mathcal{F})=\mathcal{F}_{\alpha \beta} \mathcal{F}^{\alpha \beta}, \\
\mathcal{I}_{2}=2 *(\mathcal{F} \wedge \mathcal{F})=\mathcal{F}_{\alpha \beta} \mathcal{F}_{*}^{\alpha \beta},
\end{gathered}
$$

which can be expressed in terms of the electromagnetic ones:

$$
\begin{aligned}
& \mathcal{I}_{1}=\cos (2 \alpha) I_{1}+\sin (2 \alpha) I_{2}, \\
& \mathcal{I}_{2}=\cos (2 \alpha) I_{2}-\sin (2 \alpha) I_{1} .
\end{aligned}
$$


The second auxiliary invariant vanishes if we choose $\alpha$ function to satisfy the following:

$$
\tan (2 \alpha)=\frac{I_{2}}{I_{I}}
$$

When the second invariant of a 2-form vanishes,

$$
\mathcal{I}_{2}=4 \mathcal{E} \cdot \mathcal{B}=0
$$

one says that the 2-form is decomposable (meaning that it can be written as a simple bivector) [30, 31].

However, the decomposition is not unique, as we can write the 2-form in three different ways:

$$
\begin{aligned}
& \mathcal{F}=\frac{1}{\mathcal{E}_{(1)}} \mathcal{E} \wedge\left(\mathcal{E}_{(1)} \theta^{(0)}+\mathcal{B}_{(3)} \theta^{(2)}-\mathcal{B}_{(2)} \theta^{(3)}\right)-\frac{\mathcal{E} \cdot \mathcal{B}}{\mathcal{E}_{(1)}} \theta^{(2)} \wedge \theta^{(3)}, \\
& \mathcal{F}=\frac{1}{\mathcal{E}_{(2)}} \mathcal{E} \wedge\left(\mathcal{E}_{(2)} \theta^{(0)}+\mathcal{B}_{(1)} \theta^{(3)}-\mathcal{B}_{(3)} \theta^{(1)}\right)-\frac{\mathcal{E} \cdot \mathcal{B}}{\mathcal{E}_{(2)}} \theta^{(3)} \wedge \theta^{(1)}, \\
& \mathcal{F}=\frac{1}{\mathcal{E}_{(3)}} \mathcal{E} \wedge\left(\mathcal{E}_{(3)} \theta^{(0)}-\mathcal{B}_{(1)} \theta^{(2)}+\mathcal{B}_{(2)} \theta^{(1)}\right)-\frac{\mathcal{E} \cdot \mathcal{B}}{\mathcal{E}_{(3)}} \theta^{(1)} \wedge \theta^{(2)} .
\end{aligned}
$$

Here,

$$
\begin{aligned}
& \mathcal{E}=\mathcal{E}_{(\mu)} \theta^{(\mu)}=*\left(\theta^{(0)} \wedge * \mathcal{F}\right), \\
& \mathcal{B}=\mathcal{B}_{(\mu)} \theta^{(\mu)}=*\left(\theta^{(0)} \wedge \mathcal{F}\right) .
\end{aligned}
$$

Inserting Equation (63) in the last part of Equation (72), they become as follows:

$$
\begin{aligned}
& \mathcal{E}=\cos (\alpha) E-\sin (\alpha) B, \\
& \mathcal{B}=\sin (\alpha) E+\cos (\alpha) B .
\end{aligned}
$$

Substituting Equations (25) and (26),

$$
\begin{gathered}
\mathcal{E}=\left(M, \cos \alpha+\frac{\sin \alpha}{r^{2} \sin \vartheta} N, \vartheta\right) \theta^{(1)}+\frac{1}{r}\left(M,,_{\vartheta} \cos \alpha-\frac{\sin \alpha}{\sin \vartheta} N,_{r}\right) \theta^{(2)}, \\
\mathcal{B}=\left(M,_{r} \sin \alpha-\frac{\cos \alpha}{r^{2} \sin \vartheta} N, \vartheta\right) \theta^{(1)}+\frac{1}{r}\left(M, \vartheta \sin \alpha+\frac{\cos \alpha}{\sin \vartheta} N,_{r}\right) \theta^{(2)} .
\end{gathered}
$$

Due to the vanishing of Equation (68), and the fact that $\mathcal{E}_{(3)}=\mathcal{B}_{(3)}=0$, both Equations (69) and (70) coincide:

$$
\mathcal{F}=\mathcal{E} \wedge\left(\theta^{(0)}-\frac{\mathcal{B}_{(2)}}{\mathcal{E}_{(1)}} \theta^{(3)}\right)=\mathcal{E} \wedge\left(\theta^{(0)}+\frac{\mathcal{B}_{(1)}}{\mathcal{E}_{(2)}} \theta^{(3)}\right)
$$

Since

$$
\mathcal{I}_{2}=4 \mathcal{E} \cdot \mathcal{B}=4\left(\mathcal{E}_{(1)} \mathcal{B}_{(1)}+\mathcal{E}_{(2)} \mathcal{B}_{(2)}\right)=0 \Rightarrow \frac{\mathcal{B}_{(1)}}{\mathcal{E}_{(2)}}=-\frac{\mathcal{B}_{(2)}}{\mathcal{E}_{(1)}}
$$

Now, the auxiliary 2-form $\mathcal{F}$, Equation (75), is a simple bivector. Proceeding in the same way like for the pure electromagnetic field case, it is easy to find the corresponding monad fields.

In the case when $\left|\mathcal{E}_{(1)}\right|>\left|\mathcal{B}_{(2)}\right|$, we can choose the monad field as follows:

$$
\tau_{A}=\frac{\left|\mathcal{E}_{(1)}\right|}{\sqrt{\mathcal{E}_{(1)}^{2}-\mathcal{B}_{(2)}^{2}}}\left(\theta^{(0)}-\frac{\mathcal{B}_{(2)}}{\mathcal{E}_{(1)}} \theta^{(3)}\right) .
$$

Comparing Equation (77) with Equation (A.14), one finds the velocity field of the comoving observers with the electromagnetic field in the inertial reference frame, which is given by the following:

$$
v_{A}=\frac{\mathcal{B}_{(2)}}{\mathcal{E}_{(1)}}
$$

Inserting Equation (75) into Equation (64), we have the following:

$$
F=\left[\cos (\alpha)\left(p \wedge \tau_{A}\right)-\sin (\alpha) *\left(p \wedge \tau_{A}\right)\right]
$$

where

$$
p=\frac{\sqrt{\mathcal{E}_{(1)}^{2}-\mathcal{B}_{(2)}^{2}}}{\left|\mathcal{E}_{(1)}\right|} \mathcal{E}
$$

The expressions for the electric and magnetic fields in the frame (Equation (85)) are readily obtained comparing Equation (79) with Equation (3):

$$
\begin{gathered}
\mathbf{E}_{A}=\cos (\alpha) p, \\
\mathbf{B}_{A}=-\sin (\alpha) p .
\end{gathered}
$$

Both covectors are parallel to each other; consequently, the Poynting vector vanishes in this reference frame.

In the opposite case, $\left|\mathcal{B}_{(2)}\right|>\left|\mathcal{E}_{(1)}\right|$, the auxiliary 2-form can be rewritten as follows:

$$
\mathcal{F}=*\left[\mathcal{B} \wedge\left(\theta^{(0)}-\frac{\mathcal{E}_{(1)}}{\mathcal{B}_{(2)}} \theta^{(3)}\right)\right]
$$

Now, inserting Equation (82) into Equation (64), the field tensor can be rewritten as follows:

$$
F=\sin (\alpha)\left(q \wedge \tau_{B}\right)+\cos (\alpha) *\left(q \wedge \tau_{B}\right),
$$


where

$$
\begin{gathered}
q=\frac{\sqrt{\mathcal{B}_{(2)}^{2}-\mathcal{E}_{(1)}^{2}}}{\left|\mathcal{B}_{(2)}\right|} \mathcal{B} \\
\tau_{B}=\frac{\left|\mathcal{B}_{(2)}\right|}{\sqrt{\mathcal{B}_{(2)}^{2}-\mathcal{E}_{(1)}^{2}}}\left(\theta^{(0)}-\frac{\mathcal{E}_{(1)}}{\mathcal{B}_{(2)}} \theta^{(3)}\right)
\end{gathered}
$$

Comparing Equation (85) with Equation (A.14), one finds that the velocity field of the observers comoving with the electromagnetic field in the inertial reference frame is given by the following:

$$
v_{B}=\frac{\mathcal{E}_{(1)}}{\mathcal{B}_{(2)}}
$$

The expressions for the electric and magnetic fields in the frame (Equation (77)) are readily obtained comparing Equation (83) with Equation (3):

$$
\begin{aligned}
\mathbf{E}_{B} & =\sin (\alpha) q, \\
\mathbf{B}_{B} & =\cos (\alpha) q .
\end{aligned}
$$

Clearly, both covectors are parallel to each other; consequently, there is no Poynting vector in this frame.

\section{Superposition of a Coulombian Electric Field and the Field of a Point Magnetic Dipole}

As an example of a nonpure field, the superposition of a Coulombian electric field and the magnetic field is studied here, considering the following functions:

$$
\begin{gathered}
M(r, \vartheta)=\frac{Q}{r}, \\
N(r, \vartheta)=\frac{k \sin ^{2} \vartheta}{r},
\end{gathered}
$$

for which the electromagnetic field tensor is as follows:

$$
F=-\frac{Q}{r^{2}} \theta^{(1)} \wedge \theta^{(0)}+\left(\frac{k \sin \vartheta}{r^{3}} \theta^{(1)}-\frac{2 k \cos \vartheta}{r^{3}} \theta^{(2)}\right) \wedge \theta^{(3)}
$$

From Equation (89), and using Equation (4), the electric and magnetic field covectors in the inertial reference frame represented by the monad field $\tau=\theta^{(0)}$ are as follows:

$$
\begin{gathered}
\mathbf{E}=-\frac{Q}{r^{2}} \theta^{(1)} \\
\mathbf{B}=-\frac{2 k \cos \vartheta}{r^{3}} \theta^{(1)}-\frac{k \sin \vartheta}{r^{3}} \theta^{(2)} .
\end{gathered}
$$

The electromagnetic field invariants are as follows:

$$
\begin{gathered}
I_{1}=\frac{2}{r^{6}}\left[k^{2}\left(\sin ^{2} \vartheta+4 \cos ^{2} \vartheta\right)-Q^{2} r^{2}\right], \\
I_{2}=\frac{8 k Q \cos \vartheta}{r^{5}} .
\end{gathered}
$$

From the second invariant, one can see that the electromagnetic field is of the nonpure type everywhere, except in the equatorial plane. The electromagnetic field is of the null type on the surface:

$$
k^{2}\left(\sin ^{2} \vartheta+4 \cos ^{2} \vartheta\right)-Q^{2} r^{2}=0
$$

Outside this peanut-like surface, the electromagnetic field is of the electric type, while in its interior is of magnetic type; there is no light surface, but a light ring (or a light circle) on the peanut waist, with radius $r=|k / Q|$, and only there, the field is of the pure null type; see Figure 4.

From a classical point of view, the electromagnetic field of an electron is like the one discussed here, using SI units:

$$
\begin{gathered}
k \rightarrow \frac{\mu_{0}}{4 \pi} M_{e}, \\
Q \rightarrow \frac{e}{4 \pi \varepsilon_{0}},
\end{gathered}
$$

and the corresponding light ring has a radius:

$$
r=\frac{M_{e}}{c e}=1.9 \times 10^{-13} \mathrm{~m},
$$

where $M_{e} \sim M_{\mathrm{Bohr}}=9.27 \times 10^{-24} \mathrm{~J} / \mathrm{T}$ is the electron's magnetic moment, $e=1.60 \times 10^{-19} \mathrm{C}$ is the electron's charge, and $c=2.99 \times 10^{8} \mathrm{~m} / \mathrm{s}$ is the speed of light.

In the following two subsections, we consider the velocity field of this superposition in the equatorial plane where the field is of the pure type, and outside this plane where the field is nonpure.

7.1. The Velocity Field on the Superposition's Pure Field Region. On the equatorial plane, where the field is of the pure type, the field tensor (Equation (89)) is already a simple bivector. Using the ideas of Section 4 , it can be rewritten as follows:

$$
F_{I}=-*\left[\frac{k}{r^{3}} \theta^{(2)} \wedge\left(\theta^{(0)}-\frac{r}{k / Q} \theta^{(3)}\right)\right]
$$

for $r<|k / Q|$, the monad field is as follows:

$$
\tau_{I}=\frac{|k / Q|}{\sqrt{(k / Q)^{2}-r^{2}}}\left(\theta^{(0)}-\frac{r}{k / Q} \theta^{(3)}\right)
$$




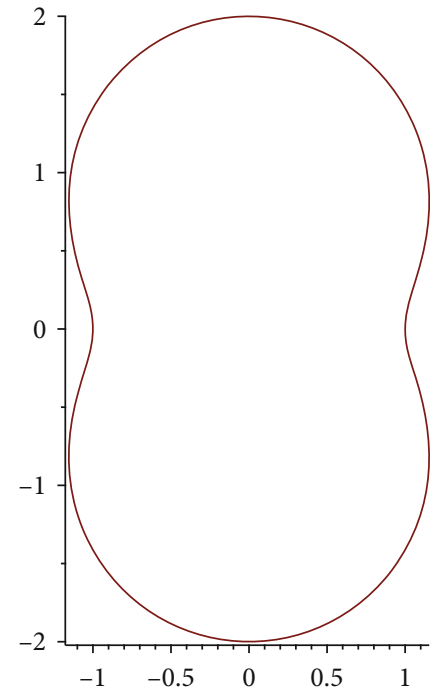

Figure 4: The displayed peanut-like surface is an implicit plot of the vanishing of the first invariant $I_{1}=0$ (Equation (92)). Inside this surface, the electromagnetic field is of the magnetic type, while outside it, of the electric type, the field is of the null type on this surface. The field is of the nonpure type everywhere, except on the equatorial plane, where it is pure $\left(I_{2}=0\right)$. Only in a circle on the peanut's waist, the electromagnetic field reaches the speed of light and there both invariants vanish, in agreement with Equation (16). Hence, there is no light surface, but a light circle.

which allows to write Equation (99) as follows:

$$
F_{I}=-*\left[\frac{\sqrt{(k / Q)^{2}-r^{2}}}{|k / Q|} \frac{k}{r^{3}} \theta^{(2)} \wedge \tau_{I}\right] \text {. }
$$

Comparing Equation (97) with Equation (3), the magnetic field in the reference frame described by the monad field (Equation (96)) is as follows:

$$
B_{I}=-\frac{\sqrt{(k / Q)^{2}-r^{2}}}{|k / Q|} \frac{k}{r^{3}} \theta^{(2)} .
$$

The field tensor (Equation (89)) can also be rewritten as follows:

$$
F_{I I}=-\frac{Q}{r^{2}} \theta^{(1)} \wedge\left(\theta^{(0)}-\frac{k / Q}{r} \theta^{(3)}\right) .
$$

In addition, for $r>|k / Q|$, we can use as a monad field:

$$
\tau_{I I}=\frac{r}{\sqrt{r^{2}-(k / Q)^{2}}}\left(\theta^{(0)}-\frac{k / Q}{r} \theta^{(3)}\right)
$$

which allows to write Equation (99) as follows:

$$
F_{I I}=-\frac{\sqrt{r^{2}-(k / Q)^{2}}}{r} \frac{Q}{r^{2}} \theta^{(1)} \wedge \tau_{I I}
$$

Comparing Equation (101) with Equation (3), the electric field in the reference frame represented by Equation (100) is as follows:

$$
E_{I I}=-\sqrt{r^{2}-\left(\frac{k}{Q}\right)^{2}} \frac{Q}{r^{2}} \theta^{(1)} .
$$

From Equation (96), inside the light surface, on the equatorial plane, the electromagnetic field is rotating rigidly in the inertial reference frame with velocity:

$$
v_{I}=\frac{Q}{k} r
$$

while, outside of the light surface, also on the equatorial plane, it is rotating differentially with velocity.

$$
v_{I I}=\frac{k}{Q r} \text {. }
$$

7.2. The Velocity Field on the Superposition's Nonpure Field Region. The auxiliary covectors are as follows:

$$
\begin{aligned}
& \mathcal{E}=-\left(\frac{Q \cos \alpha}{r^{2}}-\frac{2 k \cos \vartheta \sin \alpha}{r^{3}}\right) \theta^{(1)}+\frac{k \sin \vartheta \sin \alpha}{r^{3}} \theta^{(2)}, \\
& \mathcal{B}=-\left(\frac{2 k \cos \vartheta \cos \alpha}{r^{3}}+\frac{Q \sin \alpha}{r^{2}}\right) \theta^{(1)}-\frac{k \sin \vartheta \cos \alpha}{r^{3}} \theta^{(2)} .
\end{aligned}
$$

Inserting the corresponding components of $\mathcal{E}$ and $\mathcal{B}$ into Equations (78) and (86), we find the velocity of the comoving observers described by the monad field $\tau_{B}$ as measured by the inertial observers at rest with the rotation axis:

$v_{B}=\frac{1}{v_{A}}=\frac{Q r \cos \alpha-2 k \cos \vartheta \sin \alpha}{k \sin \vartheta \cos \alpha}=\frac{k \sin \alpha \sin \vartheta}{Q r \sin \alpha+2 k \cos \alpha \cos \vartheta}$.

The two equalities come from Equation (76). One can see that on the equatorial plane, the above expression reduces to Equation (103); hence, $v_{B}=v_{I}$. Hence, $v_{A}$ and $v_{B}$ are the velocities of the rotating observers, respectively, inside and outside the surface where the first invariant vanishes (Equation (92)).

The function $\alpha(r, \vartheta)$ is found by plugging Equation (91) in Equation (67):

$$
\alpha=\frac{1}{2} \arctan \left[\frac{4 k Q r \cos \vartheta}{k^{2}\left(\sin ^{2} \vartheta+4 \cos ^{2} \vartheta\right)-Q^{2} r^{2}}\right] .
$$

The speed field corresponding to Equation (106) is shown below on Figure 5; it can be seen that the speed of the electromagnetic field vanishes on the $z$-axis, growing to reach the speed of the light on a ring on the waist of the peanut-like surface. The white dotted line corresponds to the vanishing of the first electromagnetic invariant; inside it, the speed field for $v_{B}$ is plotted, while outside for $v_{A}$. The field is everywhere of the 


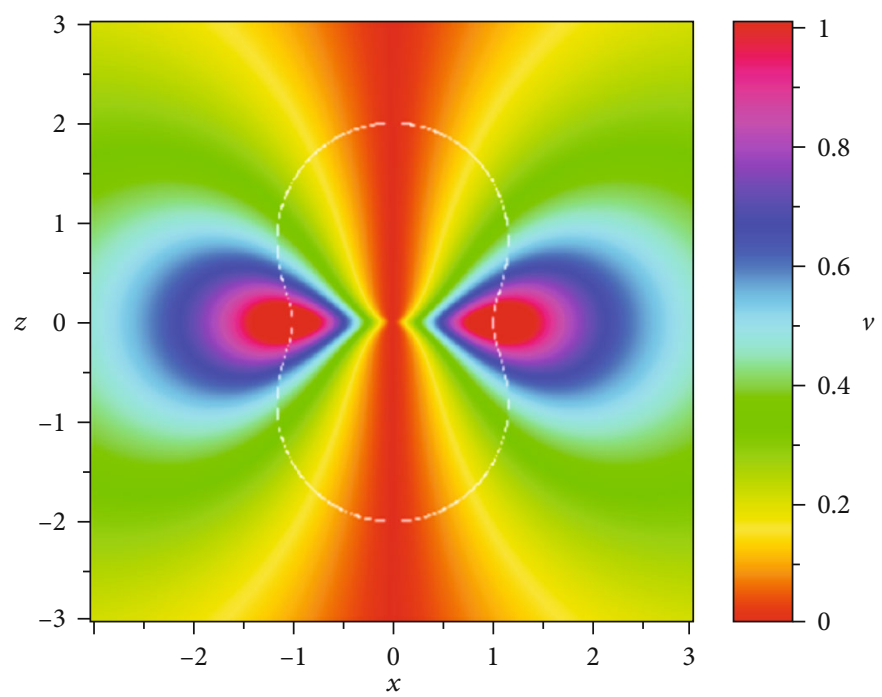

Figure 5: Speed of the observers of vanishing Poynting vector for the superposition of a Coulomb field and the magnetic field of a point dipole. With respect to the $z$-axis, the left half is approaching while the right half is receding. The white dotted line corresponds to the vanishing of the first electromagnetic invariant; inside it, the speed field for $v_{B}$ is plotted, while outside for $v_{A}$. The field is everywhere of the nonpure type, except on the equatorial plane. Only on the equatorial plane, inside the surface $I_{1}=0$, the electromagnetic field rotates rigidly. Notice that the speed of light is reached only on the light ring (the intersection of the surface $I_{1}=0$ and the equatorial plane $\vartheta=\pi /$ 2 ), where $I_{2}=0$; only there the electromagnetic field is of the pure null type; notice that this is in complete agreement with Equation (16).

nonpure type, except on the equatorial plane. Only on the equatorial plane, inside the surface $I_{1}=0$, the electromagnetic field rotates rigidly. Notice that the speed of light is reached only on the light ring (the intersection of the surface $I_{1}=0$ and the equatorial plane $\vartheta=\pi / 2$, where $I_{2}=0$ ); only there the electromagnetic field is of the pure null type; notice that this is in complete agreement with (Equation (16)).

\section{Conclusions}

In this work, a covariant $3+1$ splitting formalism was used to study rotating axially symmetric electromagnetic fields in special relativity. Two sets of observers have been introduced: a family of observers at rest with the symmetry axis and a family of comoving observers with the electromagnetic field in rotation around the symmetry axis. These comoving observers do not detect any Poynting vector in their corresponding reference frame. Due to the presence of light surfaces (or light circles), i.e., the regions where the electromagnetic field rotates with the speed of light, it was not possible to fill the entire space with one single family of rotating observers, instead two subfamilies were required, one at each side of the surface.

From a methodological point of view, to introduce the rotating observers, it was necessary to consider two cases:

(1) For pure nonnull electromagnetic fields, $I_{1} \neq 0$ and $I_{2}=0$, two subsets of rotating observers $\{I, I I\}$ have been introduced by looking for the reference frame where either the electric vector or the magnetic vector is absent

(2) For nonpure electromagnetic fields, $I_{2} \neq 0$, other two subsets of rotating observers $\{A, B\}$ have been introduced, this time by looking for the reference frame where the electric and magnetic vectors are parallel to each other

In each case, the exact expressions for these observers' velocities have been given with respect to the reference frame at rest with the symmetry axis.

In the case of pure fields, it was found that the electromagnetic field can rotate in a vast number of ways, from rigid rotation to different kinds of differential rotation. It also became apparent that for each rotational state of a magnetic field, there was a different associated electric field, although they were all similar up to a conformal factor; a different associated charge density and a different light surface. This light surface is in the regions where the electromagnetic field is of the pure null type. Several examples have been given; all of them are related to rotating magnetic dipoles, to show these latter features.

In the case of nonpure fields, the calculations have been more cumbersome. However, it has been also clear that they can also rotate in a vast number of ways. As an example, a superposition of a Coulombian electric field and dipolar magnetic field has been presented. The resulting field can display different kinds of rotation, from the rigid one to the differential one. The field's rotation speed reaches that of the light only in a circle on the equatorial plane.

As expected from Equation (16), the above examples show that only null pure fields rotate with the speed of light, while other types rotate with a subluminal speed.

The examples employed can be used not only for pedagogical purposes, but they are also of relevance in astrophysics and cosmology. In particular, these examples suggest that the magnetospheres of stars and other astrophysical objects may be quite diverse depending on the rotational state of the electromagnetic field. In the case of numerical studies of 
neutron stars and other hard objects, it is necessary to take into account that corotating frames do not describe correctly the situation on the light surface, instead it should be better to consider a reference frame at rest with the rotation axis.

As suggested by other authors $[10,11]$ and the examples presented here, the charge density in the magnetosphere of neutron stars is separated into regions of positive and negative charges. These regions offer a safe environment for antimatter to accumulate in time. Perhaps from the annihilation of this antimatter with cosmic rays, the charge density could be reconstructed. If this were the case, it would be possible to determine the rotation state of the electromagnetic field around a neutron star, in particular for those stars possessing a magnetic dipolar moment aligned with the rotation axis. As an application of this theory to the study of neutron stars and pulsars, it would be possible to get the function $d M / d N$, from Equation (49), a key ingredient of function $\xi$ (Equation (32)). This function determines the form of the light surface, $\xi=1$, Equation (35); the 4-velocity field of the electromagnetic field, Equations (36) and (41); and the structure of the electric field, Equation (46). These latter features are known so far from numerical studies of neutron stars with a rigidly corotating electromagnetic fields around them [21, 28, 29], but of course the corresponding features of the real neutron stars could be more diverse than these models suggest.

\section{Appendix}

\section{A. Cartan Formalism and Theory of Arbitrary Reference Frames}

Let us consider the four-dimensional Minkowski space with the signature $(+,-,-,-)$, although our approach works for full general relativity. The Greek indices are fourdimensional taking values from 0 to 3 , and the Latin indices are three-dimensional. The summation convention of Einstein for repeated indices is also adopted. However, in the formalism of reference frames, there are only Greek indices, and the splitting into space-like and time-like physical objects means that the first are orthogonal in their free indices to the time-like monad vector (they are projected to the three-dimensional space of the reference frame), whereas the time-like objects represent contractions with the monad vector. The indices in parentheses are tetrad components.

The line element,

$$
d s^{2}=d t^{2}-d r^{2}-r^{2}\left(d \vartheta^{2}+\sin ^{2} \vartheta d \varphi^{2}\right)
$$

together with the corresponding orthonormal tetrad,

$$
\begin{gathered}
\theta^{(0)}=d t, \\
\theta^{(1)}=d r, \\
\theta^{(2)}=r d \vartheta, \\
\theta^{(3)}=r \sin \vartheta d \varphi,
\end{gathered}
$$

is employed.
We will use the Cartan formalism of exterior forms. It provides a natural language to describe a wide range of physical theories, like electromagnetic fields [2, 3, 31], frames of reference $[15,18]$, and perfect fluids [32]. The coordinate bases are in these case sets of four covectors (1-form), $d x^{0}$, $\cdots, d x^{3}$, whereas the orthonormal tetrad base is $\theta^{(0)}, \cdots, \theta^{(3)}$. Each of these bases of 1-form is actually a 4-dimensional covector. The wedge (exterior) product is simply the antisymmetric tensor product (with the antisymmetrization denoted by the Bach parentheses). The rank of the forms can go from zero (for scalars) up to 4; the higher-rank forms are identically zero in four dimensions. The scalar product of vectors and covectors is represented by a point if the vectors are written without indices, $A \cdot B$. In the opposite case, the product will have a more general meaning, $d x^{\mu} \cdot d x^{v}=g^{\mu \nu}$ : the product of two covectors of the base gives the contravariant component of the metric tensor, with the same indices as the factors of the product.

The dual conjugation with respect to the components, i.e., in their indices, is denoted by the asterisk symbol placed above the corresponding subindexes or under the superindexes. On the other hand, the Hodge asterisk represents the dual conjugation written in a more abstract form with the asterisk placed in front of the form. It is convenient to recall that the asterisk acts onto the bases of the form being equivalent to the conjugation of the components of the form (but not both simultaneously, of course). If two Hodge asterisks are applied, then the forms of odd rank do not change, whereas those of even rank change sign.

With this definition,

$$
*\left(d x^{\alpha_{1}} \wedge \cdots \wedge d x^{\alpha_{p}}\right)=\frac{1}{(4-p) !} E^{\alpha_{1} \cdots \alpha_{p}} \beta_{1} \cdots \beta_{4-p} d x_{1}^{\beta} \wedge \cdots \wedge d x^{\beta_{4-p}}
$$

where

$$
\begin{gathered}
E_{\alpha \beta \gamma \delta}=\sqrt{-g} \varepsilon_{\alpha \beta \gamma \delta}, \\
E^{\alpha \beta \gamma \delta}=-\frac{1}{\sqrt{-g}} \varepsilon_{\alpha \beta \gamma \delta}
\end{gathered}
$$

are the covariant and contravariant components of the axial tensor of Levi-Cività, and the Levi-Cività symbol is defined as follows:

$$
\begin{gathered}
\varepsilon_{\alpha \beta \gamma \delta}=\varepsilon_{[\alpha \beta \gamma \delta]}, \\
\varepsilon_{0123}=+1
\end{gathered}
$$

By means of exterior differential operator,

$$
d:=d x^{\mu} \nabla_{\partial_{\mu}} \wedge \equiv \theta^{(\mu)} \nabla_{X_{(\mu)}} \wedge
$$

one can introduce the gradient, divergence, and rotor operators; see [15].

Any specific reference frame is physically related only with a state of motion of a swarm of probe observers together 
with their detectors defined in terms of a congruence of timelike worldlines or, in an equivalent way, in terms of $\tau$, the tangent vectors to the worldlines.

Therefore, $\tau$ is a unitary time-like vector:

$$
\tau \cdot \tau=1
$$

Moreover, the metric tensor $g$ is required as an additional ingredient to introduce the four-dimensional projector:

$$
b=g-\tau \otimes \tau,
$$

which is also used as a metric tensor in the three-dimensional subspace locally orthogonal to the field $\tau$. Since $b_{\mu \nu} \tau^{\nu} \equiv 0$, $\operatorname{det} b_{\mu \nu}=0$, and $b$ the signature of $b$ is taken as $0,-,-,-$, the "three-dimensional" scalar product of two vectors is as follows:

$$
A \bullet B=-b_{\mu \nu} A^{\mu} B^{\nu} \equiv *[(\tau \wedge A) \wedge *(\tau \wedge B)],
$$

where these vectors are projected automatically onto the aforementioned three-dimensional subspace. If the vectors belong already to the mentioned subspace, they are usually represented in bold fonts: $\mathbf{A}^{\mu}=b_{v}^{\mu} A^{v}$. The axial "threedimensional" vectorial product is as follows:

$$
A \times B=*(A \wedge \tau \wedge B)
$$

These algebraic operations are equivalent locally to the usual scalar and vectorial products; therefore, we denote them by the same symbols.

An arbitrary 4-covector $q$ has a scalar projection onto the monad:

$$
\stackrel{(\tau)}{q}:=q \cdot \tau
$$

and a vector projection onto the 3-dimensional subspace:

$$
\stackrel{(3)}{q}:=b(q, \cdot)
$$

which is orthogonal to the monad by definition. Therefore,

$$
q=\stackrel{(\tau)}{q} \tau+\stackrel{(3)}{q}
$$

The 4 -velocity $u$ of any object can be easily expressed through its 3-velocity $v$ and the monad vector, namely,

$$
\left\{\begin{array}{l}
u=\frac{d t}{d s}(\tau+v), \\
v=b\left(\frac{d x}{d t}, \cdot\right),
\end{array}\right.
$$

where physical time and length relative to the reference frame under consideration are $t$ and $x$; in particular, this means $\tau$ $=d t$; hence, $u \cdot \tau=d t / d s$, while $s$ is the proper time along the $u$ 's-world line.
The covariant derivative of the wavevector is decomposed in the form:

$$
\tau_{\mu ; \nu}=\tau_{\nu} G_{\mu}+D_{\mu \nu}+A_{\nu \mu}
$$

where $G$ is the acceleration of the reference frame:

$$
G=\nabla_{\tau} \tau
$$

$D_{\mu \nu}$, the symmetric part of space projector, is the tensor of the rate of change of the deformations, i.e., of the congruence describing our reference body:

$$
D_{\mu \nu}=\tau_{\alpha ; \beta} b_{(\mu}^{\alpha} b_{v)}^{\beta}
$$

where the parentheses denote the symmetrization. This interpretation is due to the fact that the Lie derivative with respect to the wavevector has the meaning of a time derivative and $D_{\mu \nu}$ is precisely half of the Lie derivative of the tensor projector (the metric tensor in the subspace orthogonal to $\tau$ ), and can be used as a measure of the evolution of scales in this subspace:

$$
D_{\mu \nu}=\frac{1}{2} £_{\tau} b_{\mu \nu} .
$$

This tensor can be also split in the expansion scalar, its trace:

$$
\Theta=\frac{1}{2} D_{\alpha}^{\alpha}=\frac{1}{2} D^{\alpha \beta} b_{\alpha \beta}
$$

and a traceless part which is known as the shear tensor:

$$
\sigma_{\mu \nu}=D_{\mu \nu}-\frac{2}{3} \Theta b_{\mu \nu}
$$

The antisymmetric part of the spatial projector of Equation (A.15) is the tensor of rotations:

$$
A_{v \mu}=\tau_{\alpha ; \beta} b_{[\mu}^{\alpha} b_{v]}^{\beta},
$$

where the parentheses denote antisymmetrization. Using this tensor, one can introduce the vector of rotation:

$$
\begin{gathered}
\omega=\frac{1}{2} *(\tau \wedge d \tau)=*(\tau \wedge A), \\
A=\frac{1}{2} A_{\alpha \beta} d x^{\alpha} \wedge d x^{\beta},
\end{gathered}
$$

where $*$ is the aforementioned Hodge asterisk performing the dual conjugation of the differential forms. 


\section{B. Charge and Current Densities in Rotating Reference Frames}

Consider the nonholonomic orthonormal tetrad in a rotating reference frame:

$$
\begin{gathered}
\tau=\frac{1}{\sqrt{1-v^{2}}}\left(\theta^{(0)}-v \theta^{(3)}\right), \theta^{(1)}, \theta^{(2)}, \\
\chi=\frac{1}{\sqrt{1-v^{2}}}\left(\theta^{(3)}-v \theta^{(0)}\right),
\end{gathered}
$$

where $\theta^{(\alpha)}$ are given by Equation (A.2); of course, it is defined only for $|v|<1$.

In its turn, this nonrotating tetrad can be expressed as follows:

$$
\begin{aligned}
& \theta^{(0)}=\frac{1}{\sqrt{1-v^{2}}}(\tau+v \chi), \\
& \theta^{(3)}=\frac{1}{\sqrt{1-v^{2}}}(\chi+v \tau) .
\end{aligned}
$$

Its insertion in the 4-current covector leads to the following:

$$
j=\rho \theta^{(0)}-\mathbf{J} \theta^{(3)}=\frac{1}{\sqrt{1-v^{2}}}[(\rho-v \mathbf{j}) \tau+(\nu \rho-\mathbf{J}) \chi]=\tilde{\rho} \tau-\tilde{\mathbf{J}} \chi
$$

Hence, the charge and the current densities in a rotating reference frame are as follows:

$$
\begin{aligned}
& \tilde{\rho}=\frac{1}{\sqrt{1-v^{2}}}(\rho-v \mathbf{J}), \\
& \tilde{\mathbf{J}}=\frac{1}{\sqrt{1-v^{2}}}(\mathbf{J}-v \rho) .
\end{aligned}
$$

These expressions are similar to those found in special relativity for inertial reference frames [1].

\section{Data Availability}

Figures on the manuscript show graphics of some mathematical expressions on the text.

\section{Conflicts of Interest}

The authors declare that they have no conflicts of interest.

\section{Acknowledgments}

We dedicate this work to the memory of our friend, colleague, and mentor, Professor Nikolai V. Mitskievich, who passed away on January 1st, 2019. M.A. Muñiz Torres (CVU:810197) and P.J. Domínguez (Postdoctoral fellowship No. 741231) appreciate the support provided by the Consejo Nacional de Ciencia y Tecnología (CONACyT). This work was funded by the University of Guadalajara through PROSNI program grant no. 254912/289191.

\section{References}

[1] L. D. Landau and E. M. Lifshitz, The Classical Theory of Fields, Butterworth-Heineman, 4th Edition edition, 2000.

[2] N. V. Mitskievich, "Electromagnetic fields: their classification in general relativity and propagation in a vacuum," Electromagnetic Phenomena, vol. 6, no. 17, pp. 124-132, 2006.

[3] N. V. Mitskievich, "Classification of electromagnetic fields in general relativity and its physical applications," 2008, https:// arxiv.org/abs/0802.3474.

[4] A. Rogava, G. Dalakishvili, and Z. Osmanov, "Centrifugally driven relativistic dynamics on curved trajectories," General Relativity and Gravitation, vol. 35, no. 7, pp. 1133-1152, 2003.

[5] Z. Osmanov, "Efficiency of the centrifugally induced curvature drift instability in AGN winds," Astronomy \& Astrophysics, vol. 490, no. 2, pp. 487-492, 2008.

[6] Z. Osmanov, G. Dalakishvili, and G. Machabeli, "On the reconstruction of a magnetosphere of pulsars nearby the light cylinder surface," Monthly Notices of the Royal Astronomical Society, vol. 383, no. 3, pp. 1007-1014, 2008.

[7] C. Fendt and R. Ouyed, "Ultrarelativistic magnetohydrodynamic jets in the context of gamma-ray bursts," The Astrophysical Journal, vol. 608, no. 1, pp. 378-390, 2004.

[8] L. I. Schiff, "A question in general relativity," Proceedings of the National Academy of Sciences of the United States of America, vol. 25, no. 7, pp. 391-395, 1939.

[9] D. L. Webster and R. C. Whitten, "Which electromagnetic equations apply in rotating coordinates?," Astrophysics and Space Science, vol. 24, no. 2, pp. 323-333, 1973.

[10] P. Goldreich and W. H. Julian, "Pulsar electrodynamics," The Astrophysical Journal, vol. 157, pp. 869-880, 1969.

[11] J. Petri, "Theory of pulsar magnetosphere and wind," Journal of Plasma Physics, vol. 82, no. 5, pp. 1-89, 2016.

[12] M. Zamaninasab, E. Clausen-Brown, T. Savolainen, and A. Tchekhovskoy, "Dynamically important magnetic fields near accreting supermassive black holes," Nature, vol. 510, no. 7503, pp. 126-128, 2014.

[13] G. M. Graham and D. G. Lahoz, "Observation of static electromagnetic angular momentum in vacua," Nature, vol. 285, no. 5761, pp. 154-155, 1980.

[14] J. D. Jackson, Classical Electrodynamics, John Wiley \& Sons, 3rd Ed edition, 1999.

[15] N. V. Mitskievich, Relativistic Physics in Arbitrary Reference Frames, Nova Science Publishers, Inc (US), 2006, See also this author's previous work https://arxiv.org/abs/gr-qc/9606051.

[16] N. N. Bogoliubov and D. V. Shirkov, Quantum Fields, The Benjamin/Cummings Publishing Company, Inc., 1983.

[17] V. I. Antonov, V. N. Efremov, and Y. S. Vladimirov, "Monad method and canonical formalism of general relativity," General Relativity and Gravitation, vol. 9, no. 1, pp. 9-19, 1978.

[18] G.-L. A. García-Parrado, "Dynamical laws of superenergy in general relativity," Classical and Quantum Gravity, vol. 25, no. 26pp, article 015006, 2007.

[19] R. Lanza and A. Meloni, The Earth's Magnetic Field, SpringerVerlag, Berlin-Heidelberg, Germany, 2006.

[20] J. Pétri, J. Heyvaerts, and S. Bonazzola, "Global static electrospheres of charged pulsars," Astronomy and Astrophysics, vol. 384, no. 2, pp. 414-432, 2002. 
[21] F. C. Michel and J. Li, "Electrodynamics of neutron stars," Physics Reports, vol. 318, no. 6, pp. 227-297, 1999.

[22] A. V. Tur, S. Maurice, M. Blanc, and V. V. Yanovsky, "Solutions of the plasma equilibrium within the magnetic field of a point dipole," Physics of Plasmas, vol. 5, no. 9, pp. 31013115, 1998.

[23] E. W. Hones and J. E. Bergeson, "Electric field generated by a rotating magnetized sphere," Journal of Geophysical Research, vol. 70, no. 19, pp. 4951-4958, 1965.

[24] K. Yano, The Theory of Lie Derivatives and Its Applications, North Holland Publishing Co., Groningen, 1957.

[25] T. J. Willmore, "The definition of Lie derivative," Proceedings of the Edinburgh Mathematical Society, vol. 12, no. 1, pp. 2729, 1960.

[26] A. A. Gusev, U. B. Jayanthi, K. T. Choque, G. I. Pugacheva, N. Schuch, and W. N. Spjeldvik, "Antiproton radiation belt produced by cosmic rays in the Earth magnetosphere," Geophysical Research Letters, vol. 30, no. 4, 2003.

[27] O. Adriani, G. C. Barbarino, G. A. Bazilevskaya et al., "The discovery of geomagnetically trapped cosmic-ray antiprotons," The Astrophysical Journal, vol. 737, no. 2, p. L29, 2011.

[28] C. Kalapotharakos, I. Contopoulos, and D. Kazanas, "The extended pulsar magnetosphere," Monthly Notices of the Royal Astronomical Society, vol. 420, no. 4, pp. 2793-2798, 2012.

[29] B. Cerutti and M. Beloborodov, "Electrodynamics of pulsar magnetospheres," Space Science Reviews, vol. 207, no. 1-4, article 315, pp. 111-136, 2017.

[30] B. Kosyakov, Introduction to the Classical Theory of Particles and Fields, Springer, Berlin, 2007.

[31] M. Arrayás, D. Bouwmeester, and J. L. Trueba, "Knots in electromagnetism," Physics Reports, vol. 667, pp. 1-61, 2017.

[32] N. V. Mitskievich, "Modeling general relativistic perfect fluids in field-theoretic language," International Journal of Theoretical Physics, vol. 38, no. 3, pp. 997-1016, 1999. 